\title{
Kritische Situation beim Verschluss von Skalpdefekten - und deren mögliche Vermeidung
}

\author{
Critical Situations in the Wound Closure of the Scalp \\ - How they Might be Avoided
}

Autoren

Institut

\section{Scheffka, M. Zutt}

Klinik für Dermatologie und Allergologie, Klinikum Bremen-Mitte

\section{Bibliografie}

DOI http://dx.doi.org/

10.1055/s-0034-1392432

Akt Dermatol 2015; 41: 294-299

(c) Georg Thieme Verlag KG

Stuttgart · New York

ISSN 0340-2541

\section{Korrespondenzadresse Dr. med. Daria Scheffka Klinik für Dermatologie und Allergologie Klinikum Bremen-Mitte St.-Jürgen-Str. 1 28177 Bremen Daria.Scheffka@} Klinikum-Bremen-Mitte.de

\section{Zusammenfassung $\nabla$}

Ausgedehnte Skalpdefekte stellen uns vor große operative Herausforderungen. Hohes Alter der Patienten, zugrundeliegende Komorbiditäten und auch Komedikationen, v.a. Antikoagulantien, limitieren dabei oft ausgedehnte Eingriffe. Wir möchten im Folgenden unsere Erfahrungen des Wundverschlusses am Skalp selbstkritisch darstellen und diskutieren.

Nicht zuletzt demografisch bedingt werden wir in der Dermatochirurgie zunehmend mit ausgedehnten Skalpdefekten konfrontiert, die uns nicht selten vor große operative Herausforderungen stellen. Dabei müssen wir multimorbide Patienten versorgen, denen durch Alter oder zugrundeliegende Komorbiditäten oft ausgedehnte Eingriffe nicht zumutbar sind. Immunsuppression, Antikoagulation auch mit modernen Antikoagulantien sowie ausgedehnte Feldkanzerisierung der Kopfhaut erschweren unser Handeln. Die Möglichkeiten des Wundverschlusses am Kapillitium sind häufig eingeschränkt und werden unseres Erachtens in der Literatur teilweise zu positiv dargestellt. Wir möchten im Folgenden unsere Erfahrungen auf diesem Gebiet selbstkritisch darstellen und diskutieren.

Jeder Eingriff in dieser Region sollte angepasst werden auf die individuelle Situation des jeweiligen Patienten. Neben der Flächenausdehnung des zu operierenden Befundes spielen o.g. patientenspezifische Faktoren eine entscheidende Rolle. In jedem Fall ist es empfehlenswert, sich vor Eingriffen in der Skalpregion über die Eindringtiefe von Tumoren Gedanken zu machen. Im Zweifelsfall sollte vor Eingriffen eine Bildgebung mit einer Computertomografie mit sog. „Knochenfenster“ veranlasst werden, um eine eventuelle Infiltration der Schädelkalotte auszuschließen. Gerade diese Information ist für den Operateur ausgesprochen wichtig, um eine sehr individuell an die

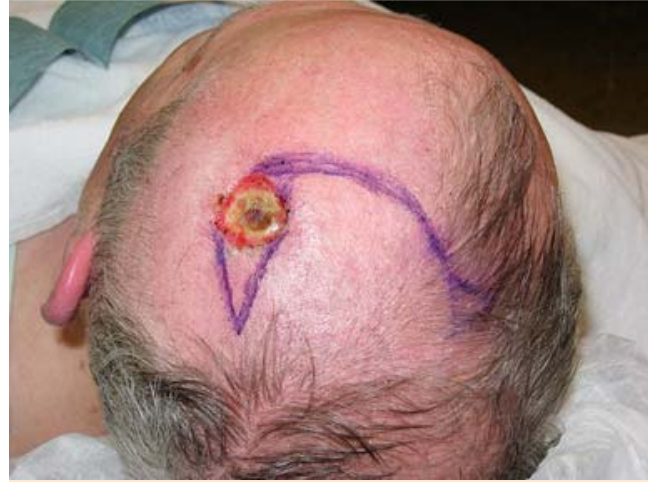

Abb. 1 Rotationslappen vor OP.

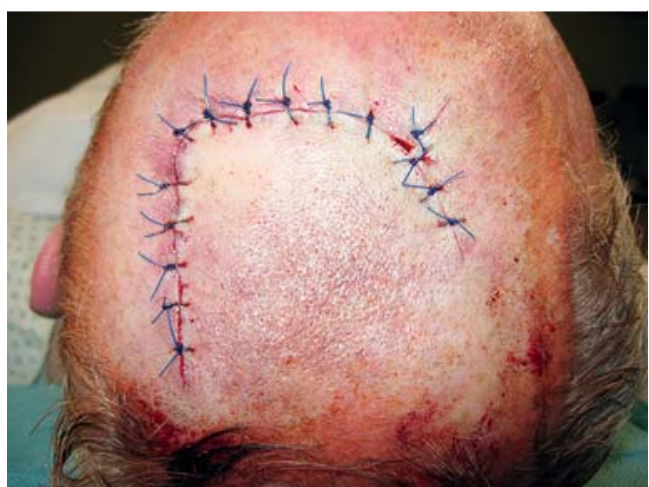

Abb.2 Komplikationsloser Verlauf des Rotationslappens.

Situation des Patienten angepasste Auswahl des zur Verfügung stehenden Verfahrens zum Defektverschluss zu treffen.

Die Dehnbarkeit der Haut am Kapillitium ist grundsätzlich durch die geringe Elastizität eingeschränkt. Trotz alledem sollte bei kleinen Defektgrößen eine Dehnungsplastik bzw. eine Nahlappenplastik im Sinne einer Verschiebelappenoder Rotationslappenplastik die Methode der Wahl sein ( $\bullet$ Abb.1, $\bullet$ Abb.2). Die Mobilisierung der Lappen muss hierbei unter der Galea aponeu- 


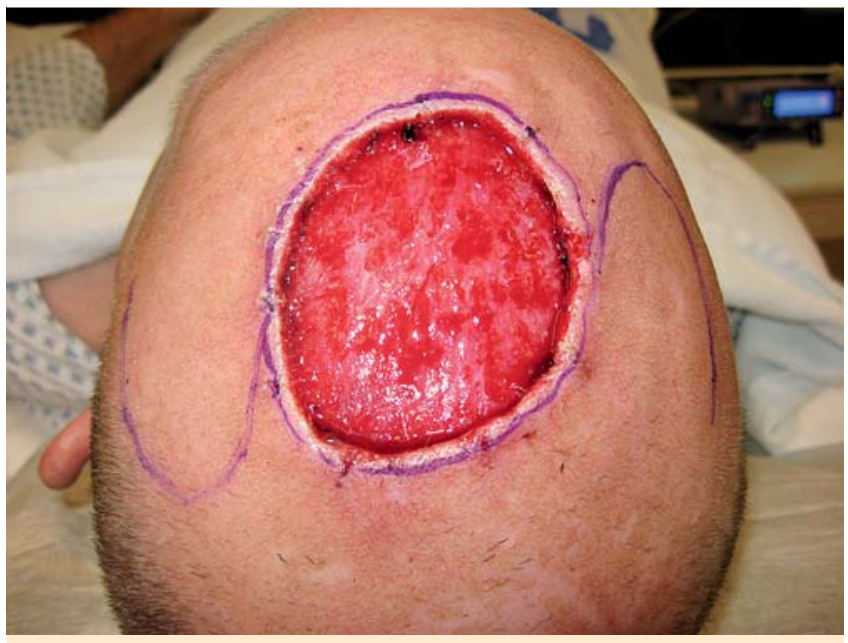

Abb.3 Komplikation Rotationslappen - Ausgangsbefund.

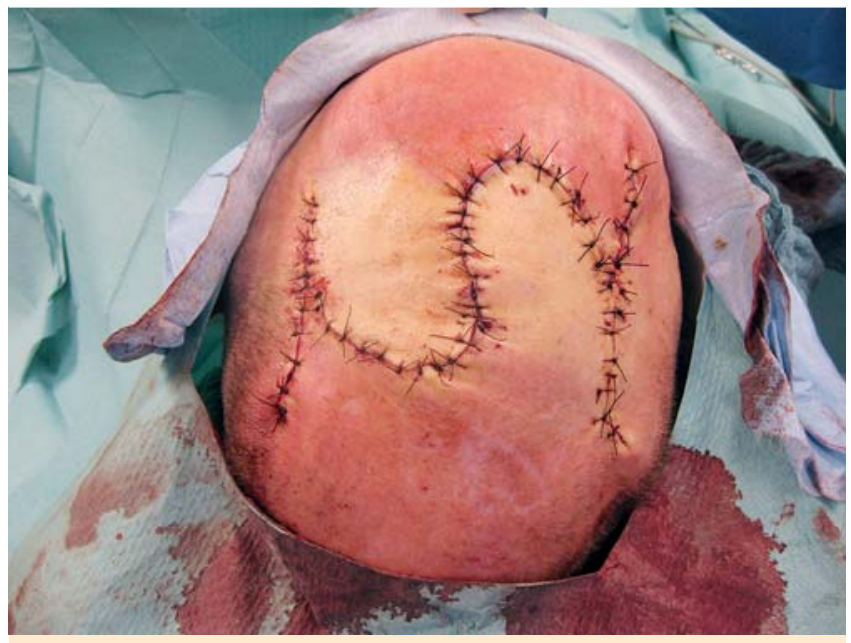

Abb.4 Komplikation Rotationslappen - unmittelbar post OP.

rotica erfolgen, welche aufgrund ihrer erhöhten Festigkeit dann auch genäht wird, wodurch sich die Zugspannung auf die Haut minimiert. Zusätzlichen Elastizitätsgewinn kann man durch jeweils ca. $1 \mathrm{~cm}$ voneinander entfernte und parallel zum Wundrand verlaufende Inzisionen der Galea erreichen [1].

Es können aber zum Teil schwerwiegende Komplikationen unter diesem Regime auftreten, wenn z.B. die Rotationsverhältnisse der Lappenplastiken nicht stimmig sind. Werden die Distanzen der Überbrückung durch Hebedefekte zu groß gewählt, können Lappenspitzennekrosen auftreten. Diese können, je nach Ausprägung, progredient sein und zu einem massiven Gewebsuntergang mit konsekutivem Austrocknen auch des Periosts führen ( $\bullet$ Abb.3-6). Daraus resultieren nicht selten monatelange Abheilungszeiten.

Insbesondere bei antikoagulierten Patienten können bei großen Lappenplastiken am Kopf relevante, revisionsbedürftige Blutungen auftreten. Aus unserer Erfahrung sollte man in der Auswahl seines Verfahrens gemäß der Prämisse „weniger ist mehr“ ein operatives Verfahren wählen, das die möglichen Komplikationen so minimal wie möglich hält. Die Ansprüche des Operateurs sollten dabei in den Hintergrund treten, nicht selten lässt sich mit einfacheren, aber vielleicht für den Operateur nicht so anspruchsvollen Eingriffen ein deutlich besseres Ergebnis erzielen.

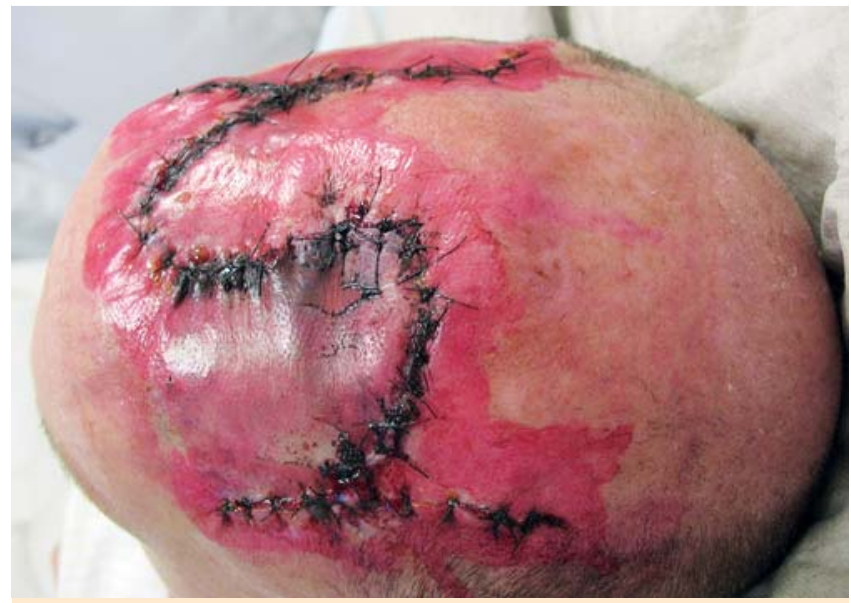

Abb.5 Komplikation Rotationslappen - ca. 5 Tage post OP mit beginnender Lappenspitzennekrose.

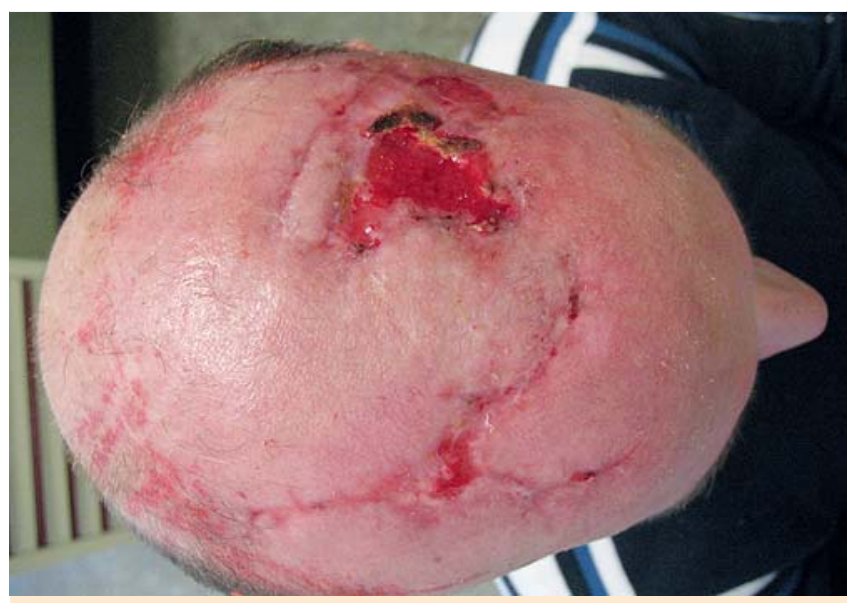

Abb. 6 Komplikation Rotationslappen - sekundäre Wundheilung nach einigen Wochen.

Die Kopfhaut lässt sich durch interne oder externe Expanderverfahren dehnen. Eine mögliche Methode ist das Einbringen eines externen Systems, bei dem Silikonschläuche im Sinne von Sekundärnahten in den Wundrand eingebracht werden. Diese sind miteinander über Kunststoffstopper verbunden. Man kann darüber über einige Tage die Wundränder durch $2 \times$ tägliches Nachspannen der Silikonbänder aneinander annähern. Eine mögliche Komplikation sind Drucknekrosen unter den Stoppern bei zu großer Spannung [2] ( $\bullet$ Abb.7-9).

"State of the art" in der Dermatochirurgie ist die zweizeitige operative Versorgung epithelialer Hauttumoren. Dabei kommt die mikrografisch kontrollierte Chirurgie zum Einsatz. Wir haben jedoch sehr gute Erfahrung mit einer einzeitigen Vorgehensweise bei alten, morbiden Patienten gemacht. Wenn man Tumoren an der Kopfhaut mit einem makroskopischen Sicherheitsabstand von einigen Millimetern klinisch im Gesunden exzidiert und in selbiger Sitzung den Defekt z. B. mit einer Hautverpflanzung verschließt, entwickeln die Patienten nur sehr selten postoperative Komplikationen. Dabei riskiert man zwar, dass die Tumoren eventuell noch schnittrandbildend sein können. Dieses Risiko nehmen wir jedoch in Kauf. Sollte dies der Fall sein, empfehlen wir eine entsprechende Nachbeobachtung oder ggf. auch eine Radiatio. Bei Basalzellkarzinomen, aber auch bei Plattenepithelkarzinomen ist dies aus unserer Sicht bei entsprechendem Alter 


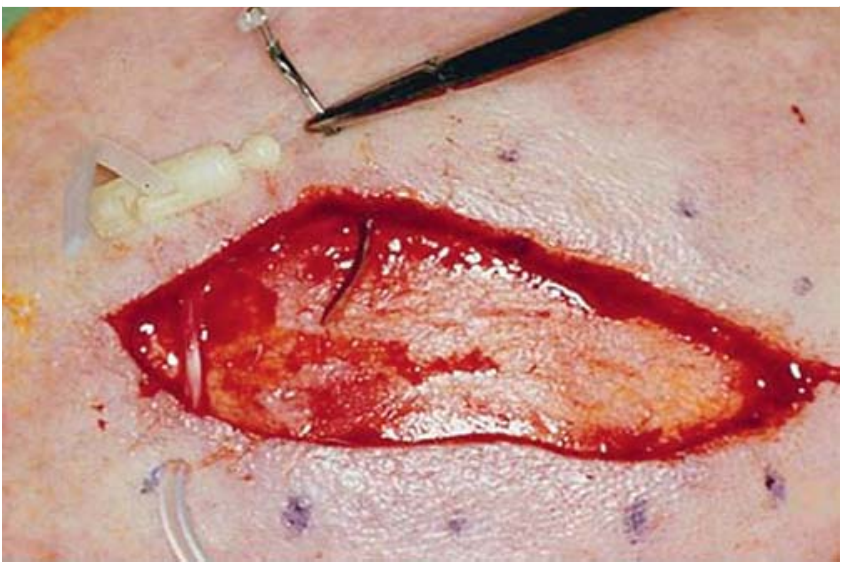

Abb. 7 Einbringen des externen Expanders. Aus: J Dtsch Dermatol 2003 [2].

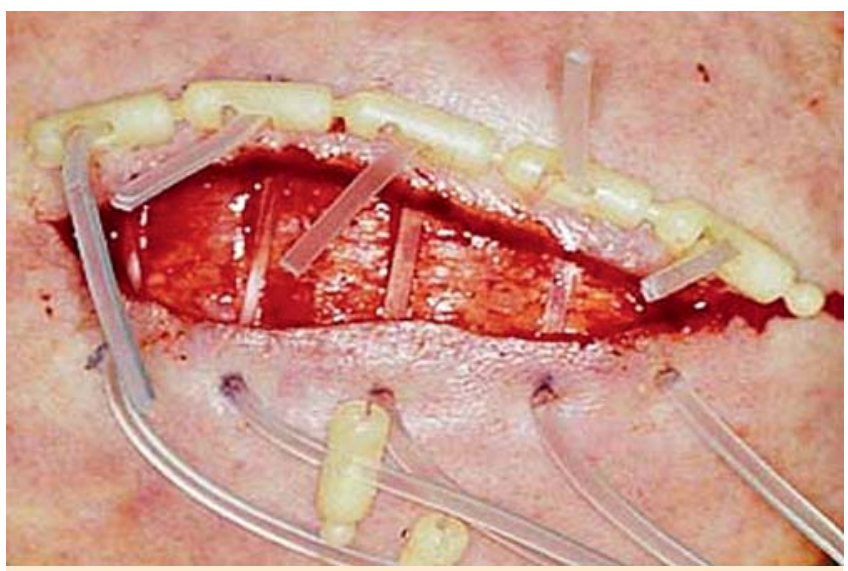

Abb. 8 Konnektion der Kunststoffstopper. Aus: J Dtsch Dermatol 2003 [2].

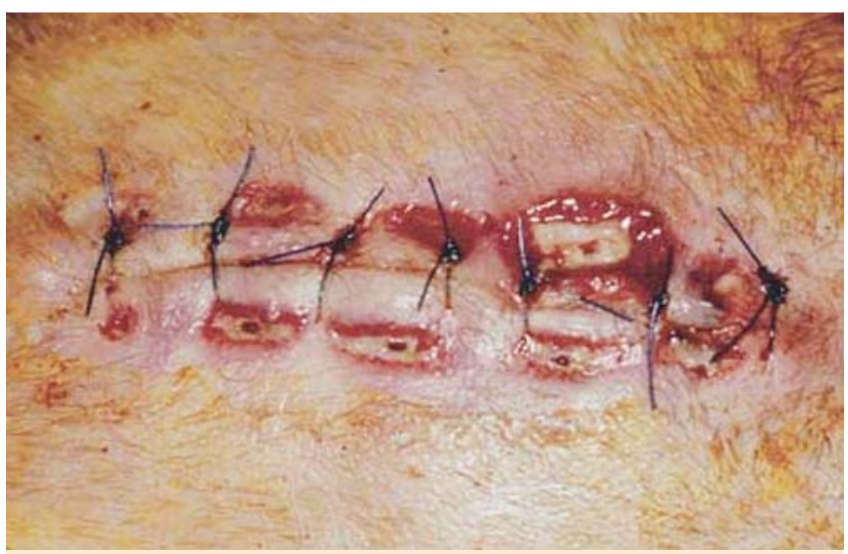

Abb.9 Drucknekrose als Nebenwirkung des externen Expanders. Aus: J Dtsch Dermatol 2003 [2].

und Komorbiditäten der Patienten zu verantworten ( $\bullet$ Abb.10, - Abb.11).

Nach erfolgreicher Granulationsstimulation auf die angestrebte Höhe wird meistens ein Spalthauttransplantat aufgebracht. Bei sehr großen Defekten (z. B. bei Feldkanzerisierung) kommt trotz ästhetischer Bedenken das Mesh-Graft-Verfahren infrage. Alternativ kann auch über eine einfache sekundäre Wundheilung nachgedacht werden, welche für den Patienten häufig die geringere Belastung darstellt, aber auch sehr zeitintensiv sein kann. Sollte man sich für eine Spalthauttransplantation als Wundverschluss entschließen, so ist die sog. retroaurikuläre Spalthaut

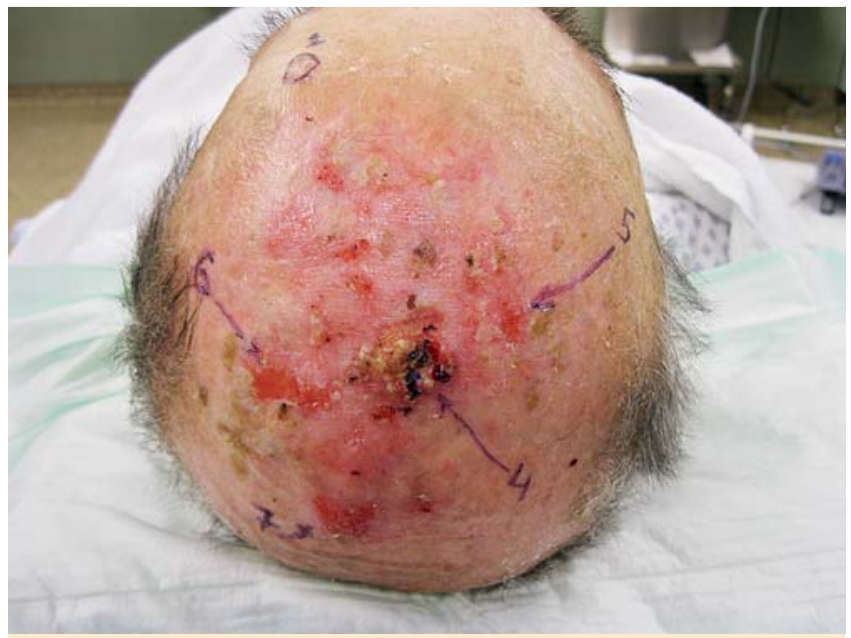

Abb.10 Ausgeprägte Feldkanzerisierung bei einem polymorbiden Patienten prä OP.

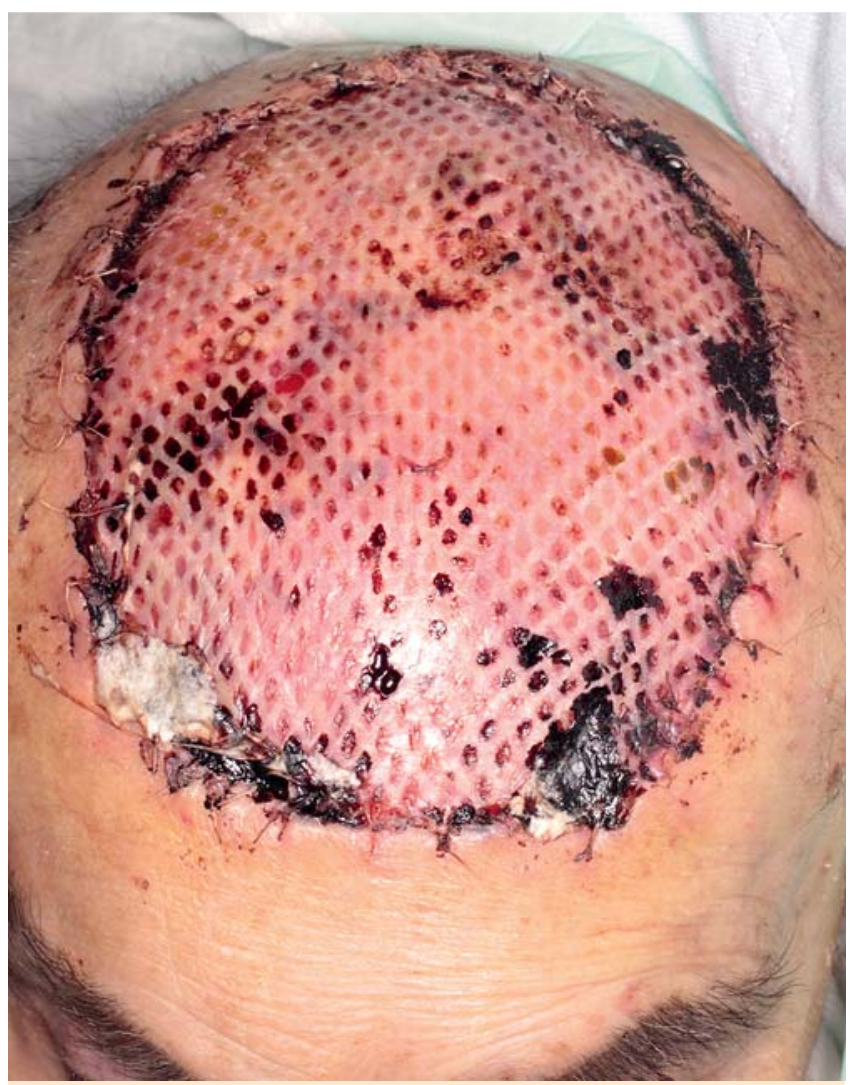

Abb.11 Z.n. einzeitiger OP der Feldkanzerisierung unter Verwendung eines Spalthauttransplantates mit hervorragendem postoperativen Ergebnis.

( $\bullet$ Abb. 12-14) ein sehr elegantes Verfahren. Bei Spalthautentnahme an anderen Donorarealen, wie z. B. am Oberschenkel ventral, entwickeln sich zum Teil sehr langwierige Abheilungszeiten. Darüber hinaus resultiert aus der Spalthautentnahme eine dauerhafte Hypopigmentierung. Entnimmt man jedoch die Spalthaut von der Kopfhaut, so heilt diese aufgrund der guten Vaskularisation sehr schnell ab. Darüber hinaus ist postoperativ keine Hypopigmentierung sichtbar, da die Entnahmestelle durch die nachwachsenden Haare überdeckt ist. Man muss präoperativ die Haare der Entnahmestelle rasieren. Das Dermatom muss entsprechend dünn eingestellt werden, damit die Haarfollikel in der 


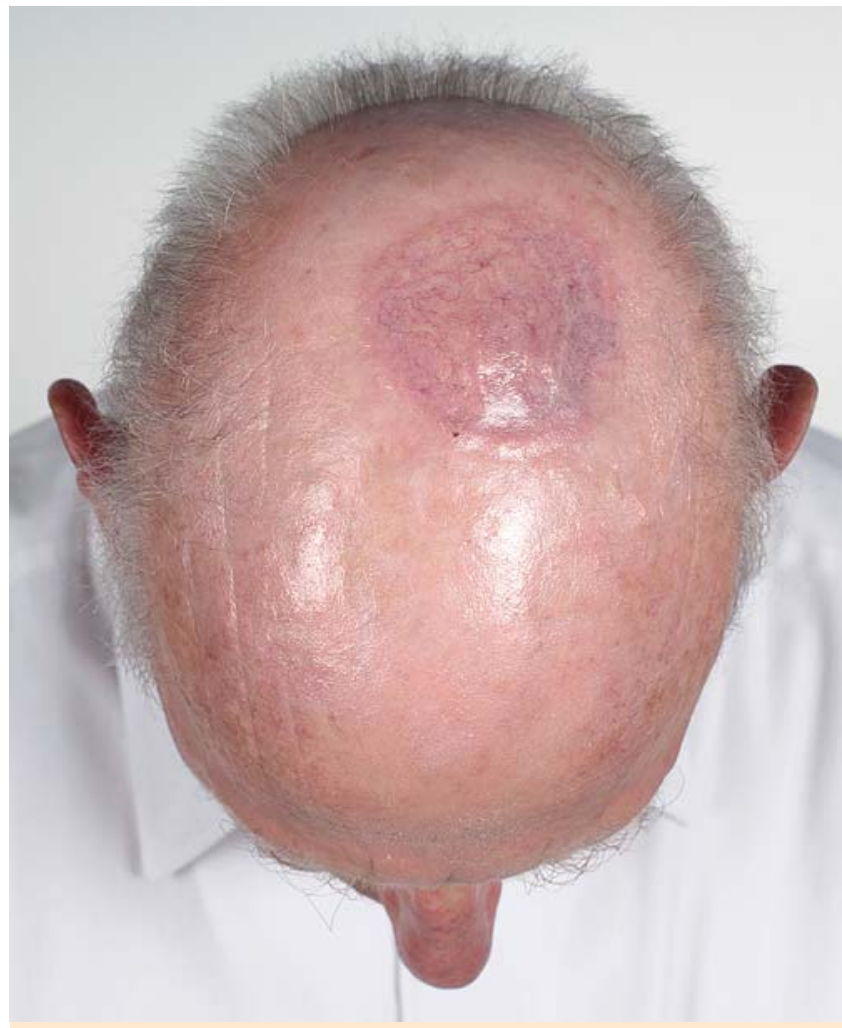

Abb.12 Gut eingewachsene retroaurikuläre Spalthaut.

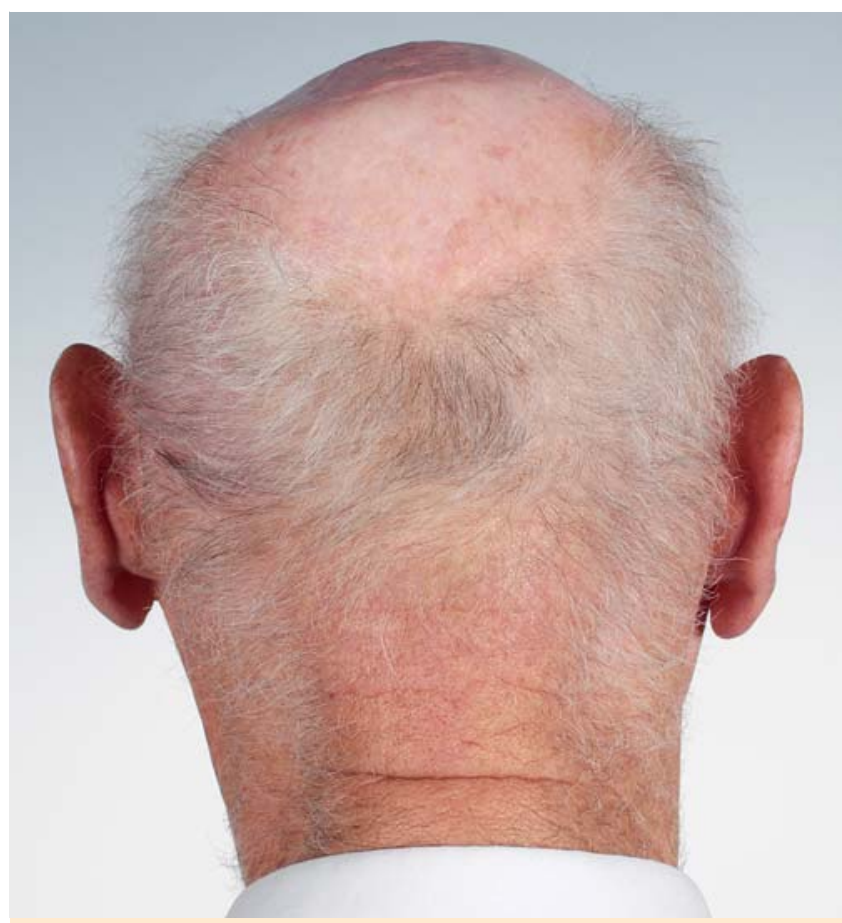

Abb.13 Keine sichtbare Entnahmestelle der retroaurikulären Spalthaut Übersicht.

Kopfhaut verbleiben. Um eine ebene Entnahmestelle zu erreichen, muss die Rundung des Schädelknochens z.B. mit der Tumeszenzlokalanästhesie geebnet werden, damit eine problemlose Entnahme der Spalthaut möglich ist. Postoperativ lässt sich die Entnahmestelle mit dem vorhandenen Deckhaar abdecken. Limitierend bei diesem Verfahren ist die Größeneinschränkung des Donorareals.

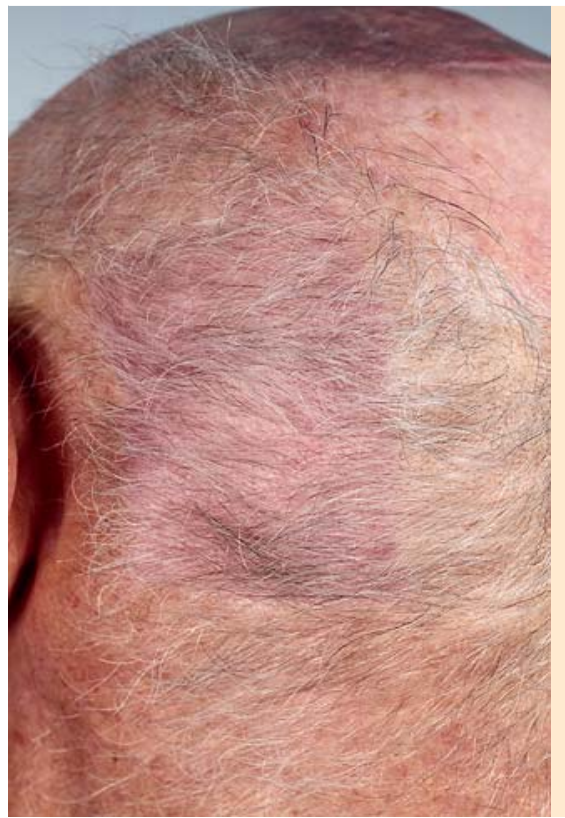

Abb. 14 Keine sichtbare Entnahmestelle der retroaurikulären Spalthaut - Detail.

Falls eine lokale Lappenplastik alleine für die Abdeckung des Defektes nicht ausreicht, lässt sich eine Kombination aus Lappenplastik und Hauttransplantation anwenden. Dabei wird der ursprüngliche Defekt mit einer Nahlappenplastik aus gesundem Gewebe verschlossen. Der dadurch entstehende neue Defekt, der ja einen gut vaskularisierten, frischen Wundgrund aufweist, wird direkt mit einem Transplantat verschlossen. Dieses Kombinationsverfahren ist sicher kritisch bei alten und morbiden Patienten zu sehen, da es doch ein aufwendiger Eingriff ist.

Sollte zunächst eine operative Versorgung des Skalpdefektes nicht möglich sein, wird unter der Voraussetzung eines intakten Periosts ein Wundgrundaufbau angestrebt, wobei zur Stimulation der Wundgranulation moderne Wundauflagen (wie z.B. Hydrokolloide) oder eine Vakuumtherapie eingesetzt werden können. Eine Vakuumtherapie kann bis zum Erreichen des angestrebten Granulationsniveaus mehrere Wochen in Anspruch nehmen. Im Falle eines kompletten Freiliegens des Knochens besteht die sehr große Gefahr der Austrocknung desselben. Um dies zu vermeiden, muss gewährleistet sein, dass mindestens $1 \times /$ Tag ein Verbandswechsel erfolgt. Dabei müssen fettige Salbengrundlagen, z.B. weiße Vaseline, zur Anwendung kommen. Diese sollten mit hochwertigen Wunddistanzgittern kombiniert werden. Dabei sind z.B. Wunddistanzgitter mit einer Silikontechnologie anzuwenden. Diese verhindern ideal ein Festwachsen am Wundgrund und ermöglichen atraumatische Verbandswechsel.

Ebenfalls bei nicht intaktem Periost (durch Tumorinfiltration oder Austrocknung) kann man Trepanationsbohrungen der Schädelkalotte mit einer Fensterung der gefäßfreien Tabula externa bis in die gefäßreiche Diploe zur Induktion von Granulationsgewebewachstum durchführen (Braunsche Knochenbohrungen). Eine solche Trepanation erfolgt mittels eines Rosenbohrers oder eines Erbium-YAG-Lasers ( $\bullet$ Abb. 15, $\bullet$ Abb. 16).

Eine elegante Methode zum Wundverschluss bei Problemwunden am Skalp ist die Verwendung von Dermisersatzprodukten. Wir konnten mit dem bovinen Dermisersatzprodukt (Integra ${ }^{\mathrm{TM}}$ ) bislang sehr gute Erfahrung sammeln. Bei einer Patientin mit einem gigantischen, exophytischen Plattenepithelkarzinom an der Stirn links mit Übergang in die behaarte Kopfhaut wendeten wir dieses Verfahren an [3] ( $\bullet$ Abb. 17). Der Tumor wurde in 


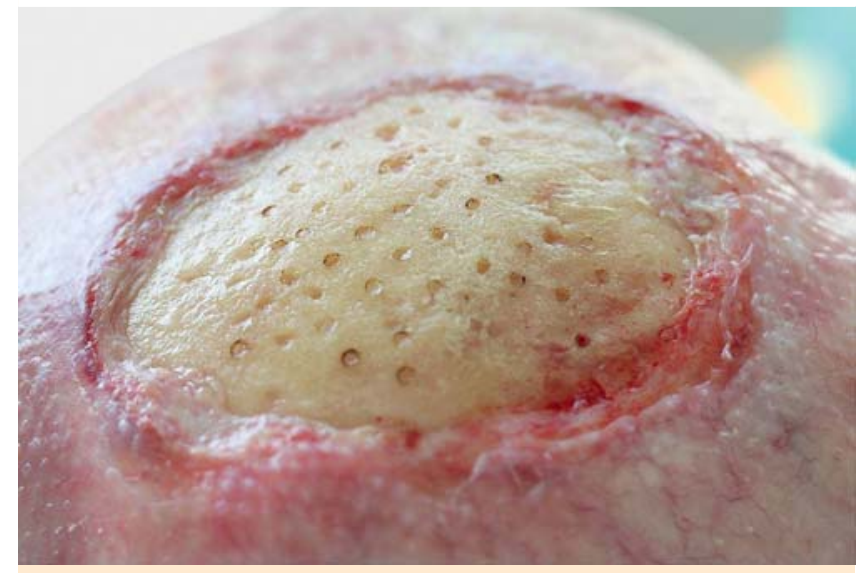

Abb. 15 Braunsche Knochenbohrungen.

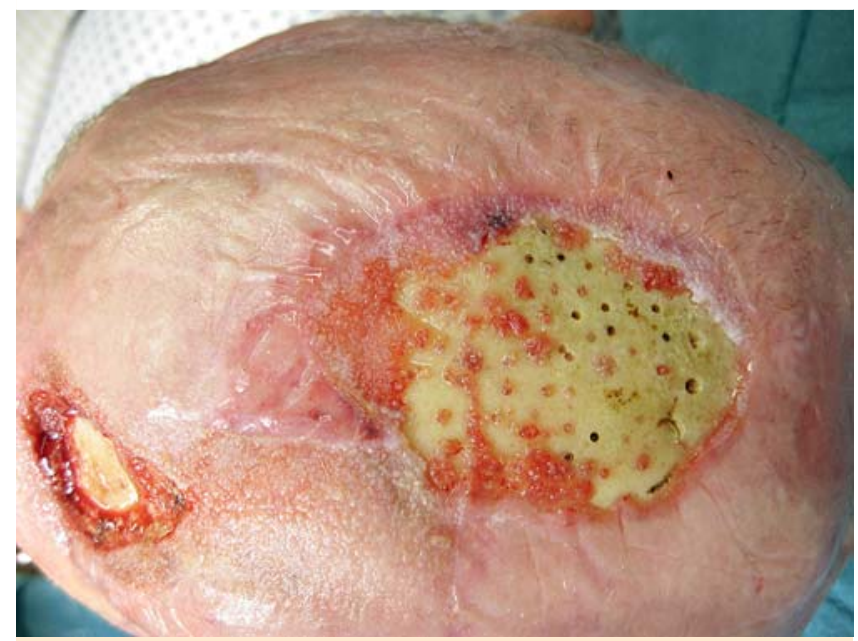

Abb.16 Braunsche Knochenbohrungen mit beginnender Granulation.

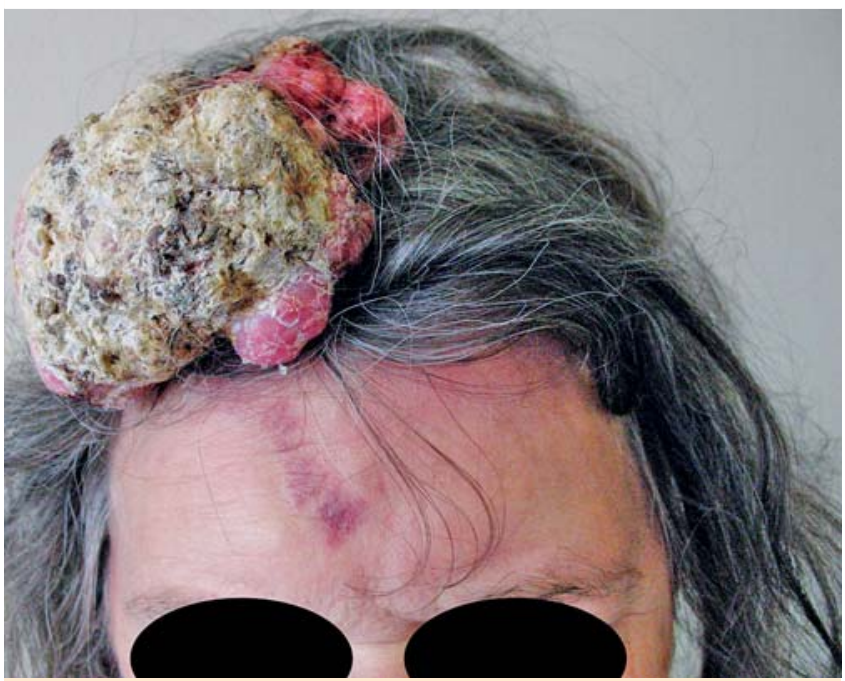

Abb.17 Gigantischer exophytischer Tumor an der Stirn links. Aus: Springer: Hautarzt 2013, Abb. 1 a [3], mit freundlicher Genehmigung von Springer Science + Business Media.

Tumeszenzlokalanästhesie radikal entfernt. Da der Tumor mit der Galea verwachsen war, erfolgte die subgaleale Exzision zur Tiefe bis zur Freilegung des Periosts des Schädelknochens. Nach erfolgreicher operativer Therapie und dem Vorliegen einer R0-Resektion verblieb ein Wunddefekt mit einer Größe von ca. $12 \times 13 \mathrm{~cm}$

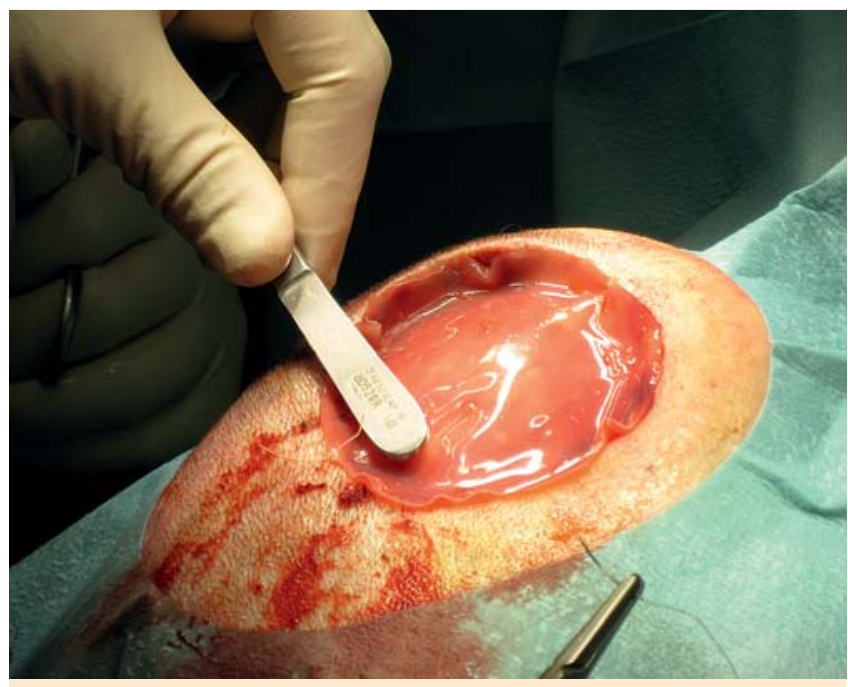

Abb.18 Annaht des Dermisersatzes. Aus: Springer: Hautarzt 2013, Abb. 4 [3], mit freundlicher Genehmigung von Springer Science+ Business Media.

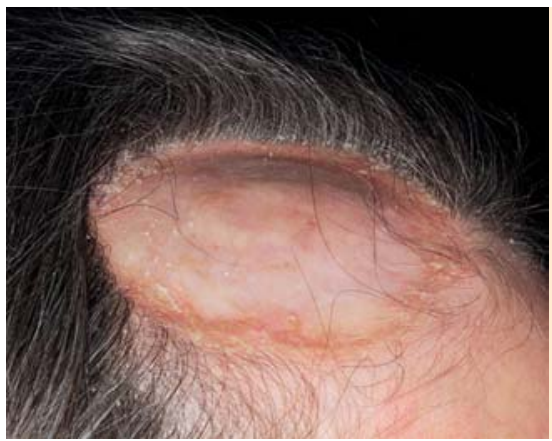

Abb.19 Befund 1 Jahr post OP. Aus: Springer: Hautarzt 2013, Abb. 8 [3], mit freundlicher Genehmigung von Springer Science + Business Media.

mit freiliegendem Periost. Zunächst wurde eine Vakuum-Therapie mit nur zögerlicher Wundgrundgranulation durchgeführt. Daher entschieden wird uns für den Einsatz des bovinen Dermisersatzes (Integra ${ }^{\mathrm{TM}}$ ). Dieser als Bilayer-Produkt verwendete kollagene Dermisersatz wurde passgenau in die Wunde eingenäht ( $\bullet$ Abb. 18). Nach insgesamt sieben Wochen war der tiefreichende Schädelkalottendefekt aufgefüllt und konnte mittels einer Spalthauttransplantation problemlos verschlossen werden. Das Transplantat heilte zu $100 \%$ ein, die Wundheilung war nach weiteren vier Wochen erfolgreich abgeschlossen. Das kosmetische wie auch funktionelle Ergebnis war sehr gut ( Abb. 19). Das Produkt besteht aus dreidimensionalem bovinem Kollagen aus Knorpel BSE-freier Rinder mit 10-15\% Glykosaminglykan (Chondroitin6-Sulfat) sowie einer Silikonfolienabdeckung. Die Silikonfolie wirkt gegen Austrocknen des Präparates. Es muss passgenau auf die Wundfläche zugeschnitten werden. Der Wirkmechanismus ist letztlich ungeklärt; es erfolgt ein Umbau des Dermisersatzproduktes in ca. drei Wochen in körpereigenes Gewebe. Dabei kommt es zur Ausbildung einer dreidimensionalen Neodermis mit Einwanderung von Fibroblasten, Makrophagen und Bildung von kapillarreichem Granulationsgewebe. Das Produkt kann direkt auf den Knochen, auch ohne Periost, aufgelegt werden. Die Vorteile dieses Verfahrens sind die einfache Anwendung sowie die Tatsache, es auch bei älteren, multimorbiden Patienten anwenden zu können. Man kann auch direkt auf das frisch eingenähte Dermisersatzprodukt eine Spalthauttransplantation anlegen. Unbedingt sollte die Anwendung von jodhaltigen Desinfektionsmitteln vermieden werden, da Jod die Kollagenmatrix des Produktes auf- 


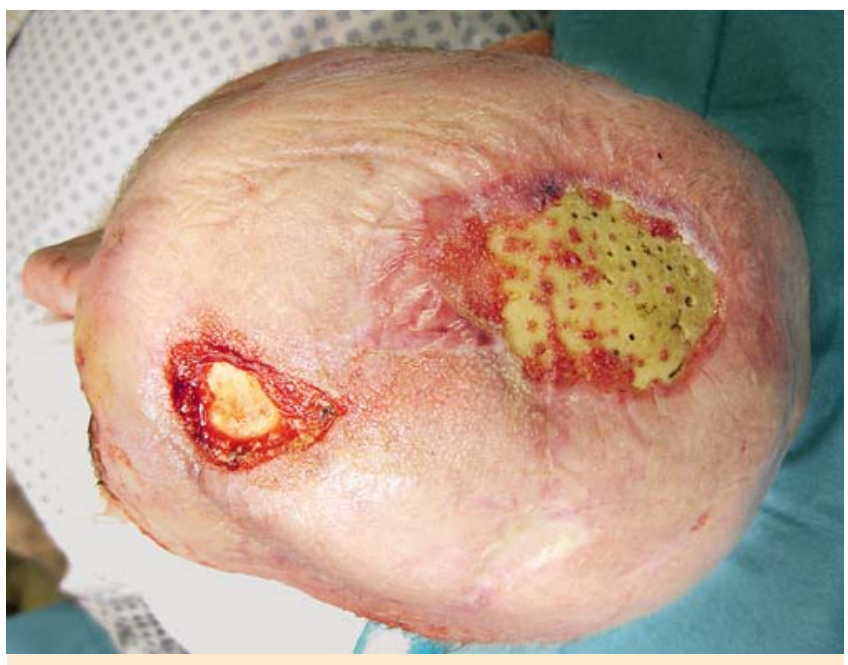

Abb. 20 Komplikationen unter Immunsuppression.

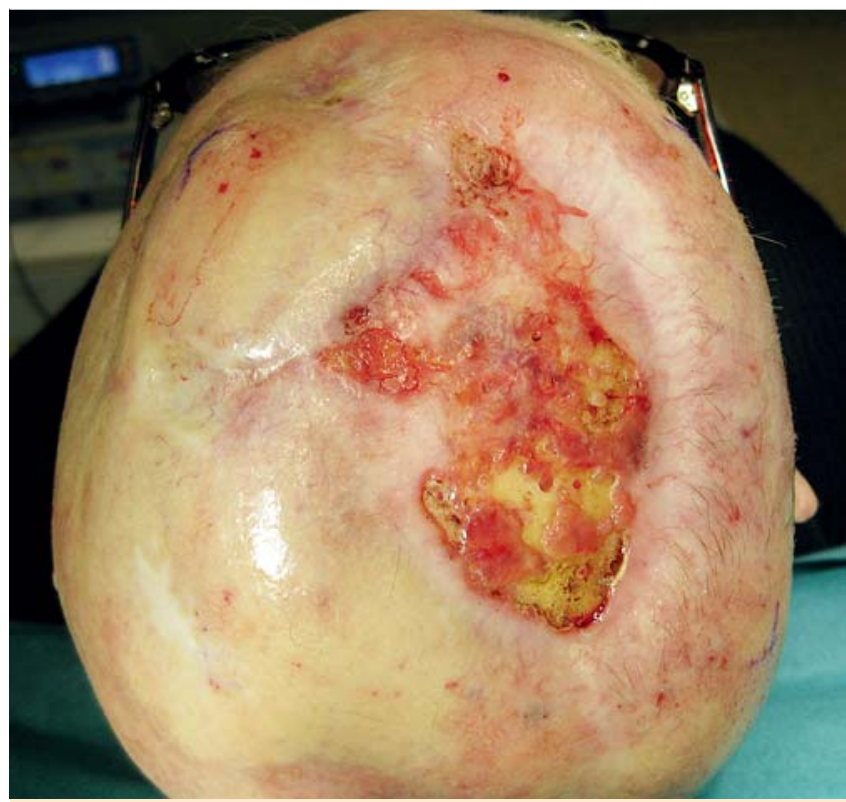

Abb. 21 Komplikationen unter Immunsuppression.

lösen kann. Ein weiterer Nachteil des Verfahrens ist der relativ hohe Preis und mögliche Wundinfekte [3].

Anhand eines Fallbeispiels aus unserer Klinik lassen sich die Schwierigkeiten aus der täglichen Praxis hervorragend demonstrieren ( $\bullet$ Abb.20, $\square$ Abb.21): Bei diesem Patienten ist nahezu die gesamte o.g. Therapiepalette angewendet worden. Es handelt sich um einen älteren, nach Nierentransplantation immunsupprimierten Patienten mit ausgeprägter Feldkanzerisierung am Kapillitium. Bei ihm wurden in den letzten Jahren zahlreiche dermatochirurgische Eingriffe bei multiplen Plattenepithelkarzinomen der Kopfhaut durchgeführt. Daher imponierte seine Kopfhaut wie ein unverschieblicher „Flickenteppich“. Bei uns wurde ein bis auf den frontoparietalen Knochen reichendes Plattenepithelkarzinom offen exzidiert. Der daraus resultierende Defekt hatte einen Durchmesser von ca. $12 \mathrm{~cm}$. Wegen der Defektgröße und der umgebenden Hautqualität war eine lokale Lappenplastik nicht durchführbar. Auch eine freie mikrozirkuläre Lappenplastik war aufgrund der bereits mehrmals operativ veränderten Gefäßverläufe nicht zu diskutieren. Schließlich begannen wir unsere postoperative Wundversorgung mit Trepanationsbohrungen und Vakuumtherapie. Trotz großen zeitlichen und finanziellen Aufwandes kam es auch nach mehreren Monaten lediglich zu einzelnen kleinen Granulationsinseln. Daraufhin wechselten wir zu einem Wundmanagement mit modernen Wundauflagen. Hierunter kam es zu einem hoffnungsvollen Granulationsschub, der jedoch bald zum Stillstand kam. Der Defekt hatte sich nach einem Jahr auf ca. $7 \times 7 \mathrm{~cm}$ der freiliegenden Schädelkalotte verkleinert. Daraufhin wurde ein bioinduktiver und bioaktiver Hautersatz eingesetzt. Die Neo-Dermis besteht aus Hyaluronsäure. Trotz antibiotischer Abschirmung kam es zu einer bakteriellen Infektion, wodurch der Defekt sich nur geringfügig verkleinerte. Ein anschließend aufgebrachtes Spalthauttransplantat wurde nach wenigen Wochen wieder abgestoßen.

Nach insgesamt ca. 18-monatiger Behandlung ist es uns trotz jeweiliger Anfangserfolge leider nicht gelungen, den Hautdefekt mit freiliegender Schädelkalotte vollständig zu verschließen. Dieses Ergebnis ist auch in Anbetracht der sehr guten Compliance des Patienten ernüchternd. Während der Behandlungsdauer wurden parallel mehrere neu diagnostizierte Plattenepithelkarzinome am Kapillitium exzidiert, was die Wundheilung immer wieder inhibierte.

Zusammengefasst ist der Wundverschluss am Skalp ein anspruchsvolles Gebiet der Dermatochirurgie. Aufgrund drohender, zum Teil schwerwiegender Komplikationen sollte man sein Verfahren den Komorbiditäten der Patienten anpassen. „Einfache“ Wundverschlüsse sind komplizierten Nah- oder Regionallappenplastiken vorzuziehen.

\section{Interessenkonflikt \\ $\nabla$}

Die Autoren geben an, dass kein Interessenkonflikt besteht.

\section{Abstract}

\section{Critical Situations in the Wound Closure of the Scalp - How they Might be Avoided \\ $\nabla$}

Large defects on the scalp need great effort to perform surgical wound closure. High age, comorbidities and comedications, like anticoagulation, are limiting interventions. We describe and discuss critically our experiences in this field.

\section{Literatur}

1 Raposio E, Santi P, Nordström RE. Effects of galeotomies on scalp flaps. Ann Plast Surg 1998; 42: 17-21

2 Zutt M, Beckmann I, Kretschmann L. Evaluation of the external tissue expander (ETE) in secondary wound closure. J Dtsch Dermatol 2003; 1: $711-715$

3 Zutt M, Ischebeck S, Kaune KM. Gigantisches Plattenepithelkarzinom des Kopfes - erfolgreiche operative Therapie und Einsatz eines bovinen Dermisersatzes. Hautarzt 2013; 64: 923 -926 